\title{
Simulating Drug Discoveries
}

\author{
Anokh Ambadipudi ${ }^{1}$ \\ ${ }^{1}$ Affiliation not available
}

July 7, 2021

Using Computer Models to Identify Synthetic Quorum Sensing Inducers for S. aureus Bacteria

By: Anokh Ambadipudi

Word Count: 4630

\section{INTRODUCTION}

The last decade has seen an increase in the implementation of computers in the biomedical field. Novel topics such as precision medicine and bioinformatics are heavily reliant on the use of modeling software in order to add a level of certainty to their results in labs. With the growing field, many prominent experiments now require some sort of proof using computer models in order to gain funds for any project they mean to complete. The use of molecular modeling stems from the study of molecular mechanics and has also been put into the form of graphing software [11]. By quantifying the different molecular forces and converting them into the software characteristics of java and python, researchers are able to understand how molecules and receptors interact before even conducting a laboratory experiment[15]. The future of biomedical engineering and research is now found in computers and as technology progresses, the accuracy of these computerized models will improve with it.

This project focuses on the use of simulation processes to understand and quantify the compatibility of different ligands with a specific receptor. Using methods found on the modeling software Schrodinger [20], different ligands can be instated to bind to the agr receptor of $S$. aureus and a number will be calculated that tells how strong the binding is. This will give a good idea to how exactly the bacteria will react in a live setting to these molecules and if these molecules do the job they are hypothesized to do. Chemical modeling has revolutionized the methodology in which scientists proceed with testing as it has benefitted the accuracy of their results. This project will have a focus on using Schrodinger's GLIDE docking software to run the in silico steps in discovering a compound that will target the quorum sensing inducers in Staph bacteria, effectively dismantling infections.

\section{LITERATURE REVIEW}

\section{Quorum Sensing}

In order to comprehend the mechanisms being tested through this project, it is crucial to understand the terminology that spans the entirety of this experiment. The question of which synthetic quorum sensing inducer could be used to stop the creation of biofilm is a complicated one, but once the context is understood, the experimentation is relatively straightforward. Quorum sensing (QS) is the regulation of gene expression in response to fluctuations in cell-population density[1]. QS is a type of primitive communication found in most prokaryotes where certain autoinducers(AIs) are used by the cell population in order to share information or express certain genes found in the colony. The AIs are produced by the bacteria in accordance with the cell population density around them. The more cells are introduced to the population, more AIs are produced 
by the bacteria[2]. Once the population reaches a high cell density, the AIs are now in enough concentration to commence some sort of cooperative cell mechanism[2]

The process in which these AIs alter the bacterial population is slightly different for every bacteria but the main sequences are nearly identical. Once the AI is produced it is let out into the extracellular matrix. It then binds to a protein receptor found on another cell and instigates an energy signaling chain, whether that be through an operon or kinases. The binding element is dependent on the types of bacteria that are being observed. Once the signaling chain reaches the genetic material of the bacteria, a specific gene is translated to form some sort of molecule or protein. Figure 1 gives a good representation of the complex QS mechanism and how different cell densities can affect the expression and production of some genes.

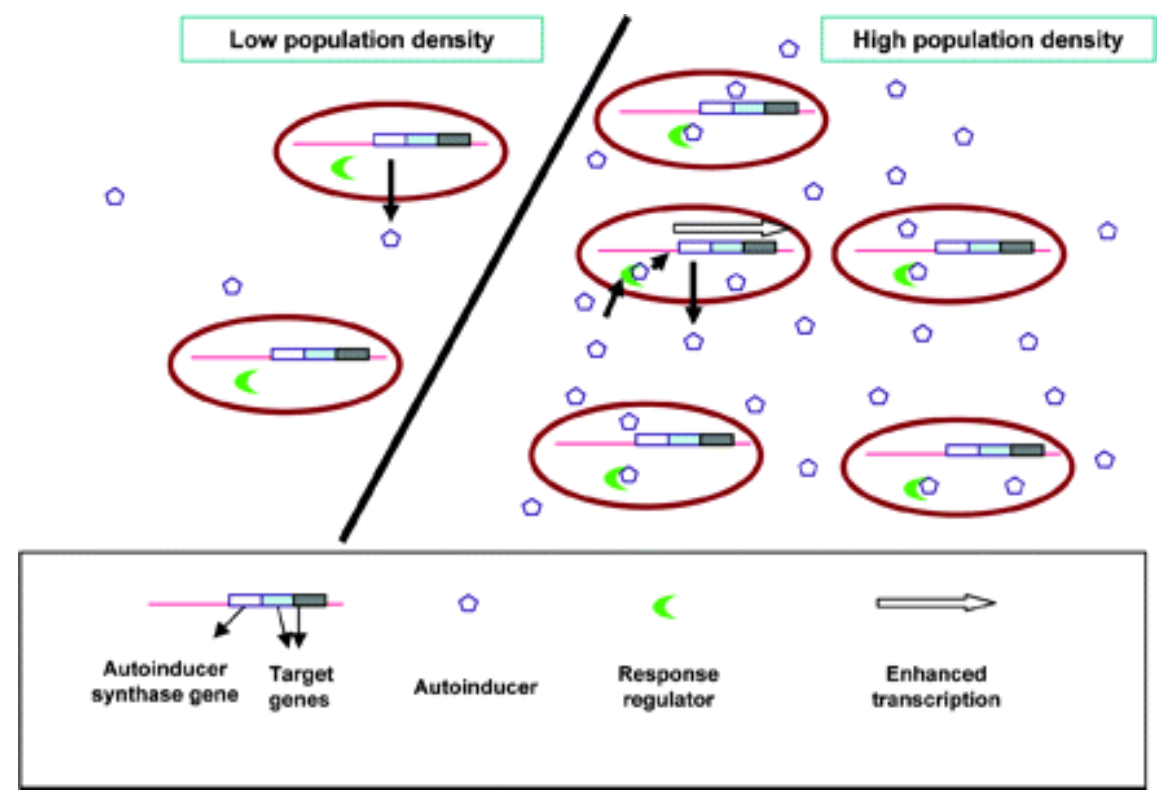

Figure 1: the relation of density to the concentration of Autoindcuers in a cellular environment. As the cellular population goes up, the amount of autoinducers in the environment increases, and with it comes an increase in protein creation through the operon the AI controls [3,4].

With the RNA coded from the QS mechanism, the bacteria can then indulge in a number of activities, the one with the most importance is the production of virulence factors. For context, virulence factors are certain chemicals released from biofilm that are a danger to the host. Virulence factors can do anything from destroy host tissue, or attack immune response to the bacteria's presence. American biologist Theobard Smith's probability of disease equation is strong evidence that toxins and virulence factors are only useful in masses[4]. Therefore, there is a necessity for high cell density through colonies known as biofilms and active quorum sensing mechanisms in order for an infectious bacteria to spread through its host.

\section{Biofilms}

In order for quorum sensing to function efficiently, bacterial cells must congregate in large masses, known as biofilms. Biofilms are a formation of cells along some sort of surface that allows for increased survivability rates for the cell population. When bacteria form in biofilms, QS is a key method of communication to express the genes that allow for defense against antibodies or bacteriophages. The AI's activate a kinase that expresses the gene to stiumualte creation of branches to reach out and physically connect to neighboring cells(figure 2). The life cycle of a quorum sensing mediated biofilm goes as follows: adhesion, maturation, and dispersion[5]. In the adhesion phase, individual cells form bonds between one another to create a web of intricate cells that use QS to communicate along the surface. The maturation phase involves the mass 
production of proteins through gene expression that help the biofilm grow and thrive in the surface it has attached to, whether living or non living. Lastly, the dispersal of the biofilm is mediated once the cells find other regions to colonize and find more nutrients to consume(fig 2).

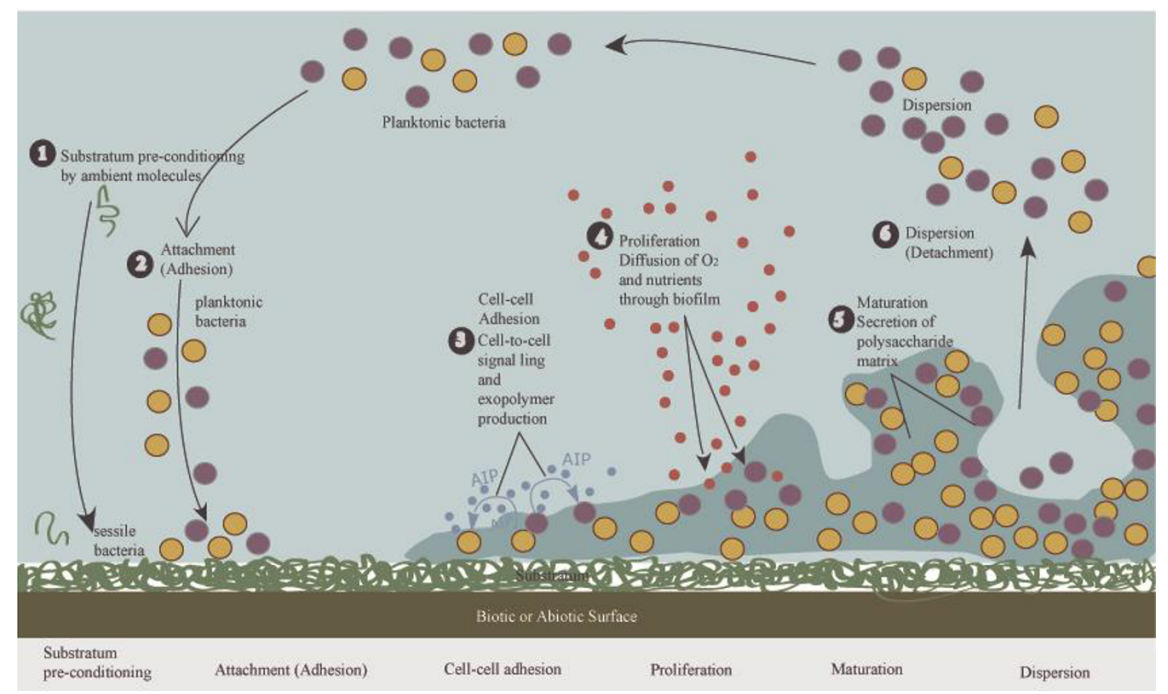

Figure 2: Life cycle of standard biofilms that are responsible for the spread of infection in a host. The bacteria first attaches to the surface (1-2) then grows in the maturation phase (3-5), and finally disperses once nutrients is low (6) [5].

Studies have shown that the release of virulence factors occurs at the peak of the maturation stage when cell density is the highest [6]. Since, the process of biofilms is completely dependent on how well the communication between bacteria is; this indirectly related the virulence of a bacterial infection to the effectiveness of quorum sensing[14].

\section{Research and Problem}

Most research has now been directed to constraining the biofilm matrix in any way possible to limit and even stop the infection. Finding alternative methods of curing bacterial infections is a hot topic in the field of disease biology and there already has been a handful of research done in regards to the role of quorum sensing in disease biology. But, most of the research done has been centered around gram negative bacteria. As stated before, quorum sensing mechanisms vary based on the type of bacteria that is being studied. The types are broken down into two groups based on the toxins each produce, gram positive bacteria and gram negative bacteria. The main difference focused on between a gram negative and gram positive bacteria lies in the type of virulence factor released by the cells. Gram negative bacteria release mainly endotoxins while gram positives are more known to release exotoxins, the former being more deadly even in small amounts[7]. Research from multiple studies shows that most gram negative bacteria use acylated homoserine lactones (AHL) while gram positive bacteria tend to use different types of oligopeptides as inducers, which are known as autoinducing peptides (AIP). Since gram negative bacteria tend to be more deadly, there is a lot more focus in creating alternative methods in terminating them. The most prominent method of manipulating the QS mechnaims was a process labelled as quorum quenching, in which synthesized enzymes (quorum quenching molecules) are used to degrade AHL signaling molecules and effectively inhibit the biofilm formation[13]. Other researchers from Oxford have shown the effectiveness of using AHL mimics acting as competitive inhibitors for the receptor, eventually halting the growth of the biofilm and temporarily stopping infections[8]. Other studies have shown how QS mediated systems, like bioluminescence in $V$. fischeri, can be controlled and manipulated[11]. 
Since most studies done have had a focus on gram negative bacteria, this experiment takes a look at the gram positive bacteria and the information that can be learned from them. First, it must be taken into consideration that the QS mechanisms in each type of bacteria are structured differently. While gram negative bacteria have the AI interact directly with the operon, gram positive use signaling cascades to allow expression of the gene. The sensor kinase signal in gram positive cells was adaptive in experiments using inhibitors and did not suppress the signaling molecule production for long enough to halt the spread of the biofilm. So now the biggest problem is finding a new method of halting biofilm production. The hypothesis created through looking at the research already done is, finding a chemical mimic of the signaling AIP and speeding the phases of the biofilm until it reaches the dispersal phase where it is no longer a threat. It is already known that the virulence factor will not show up until the peak of the maturation period so in order to end the biofilm, it could be possible to increase the rate at which the biofilm matures through the use of synthesized inducers.

The specific type of bacteria used in this project was a gram positive strain of Staphylococcus aureus. Since gram positive bacteria tend to be less hostile to patients, they tend to be neglected when examining new therapeutic prospects. One of these gram positive bacteria, staph aureus, has grown increasingly resistant to antibiotics such as methicillin. So much so that hospitals now need to classify whether a patient has a regular staph infection or MRSA (methicillin resistant staph infections). There has been a drastic increase in antibiotic resistant staph infection hospitalizations $[3,12]$ over the past decade and I believe that a solution to MRSA infections might be found in QS mechanisms. A study published by the American society of bacteriology has shown direct correlation between QS and the maturation of biofilm in staphylococcus aureus; "QS via the agr system works to regulate $S$. aureus biofilms"[9]. The agr system(figure 3) uses an autoinducer known as the autoinducing peptide(AIP) to bind with the agrC binding site which then triggers the transcription of the agr operons.

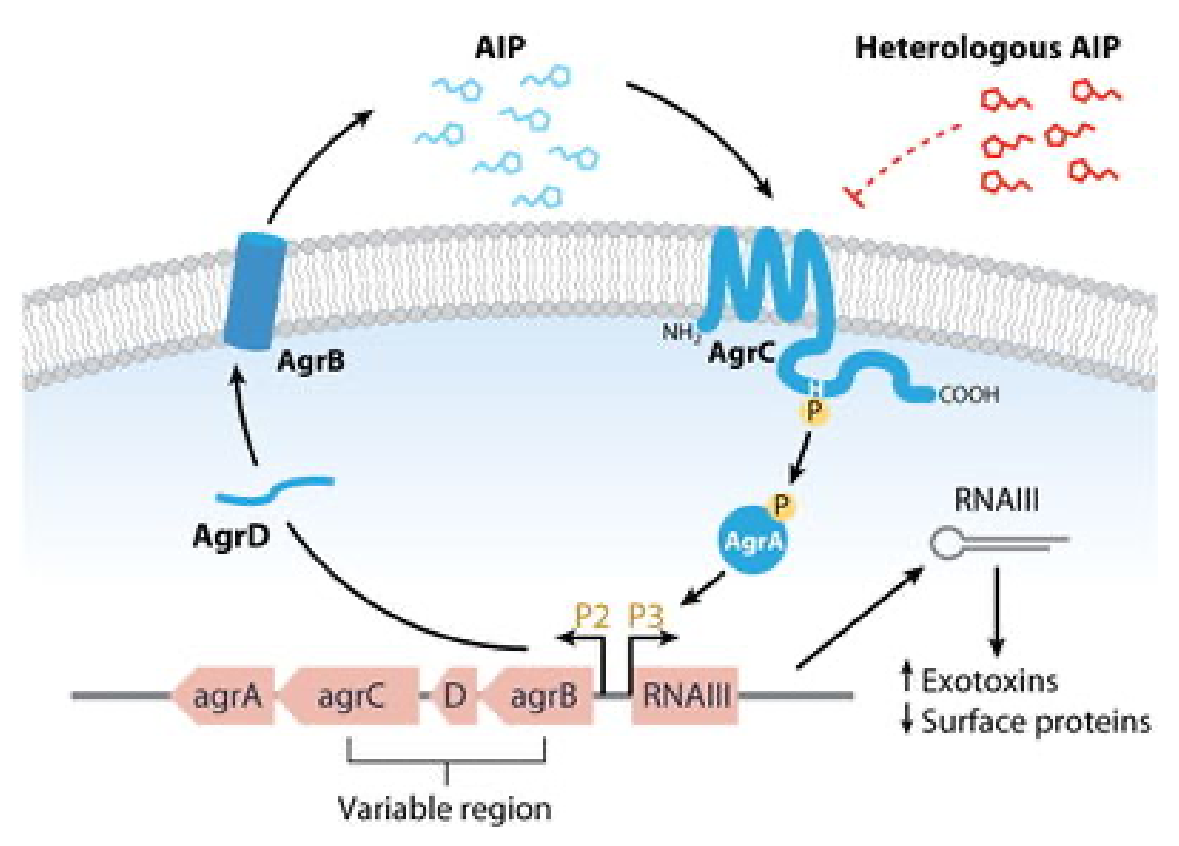

\section{R Annu. Rev. Genet. 42:541-64}

Figure 3: The receptors and operons for the QS mechanisms of S. aureus. The AIP compound binds to 
the agr-C and agr-D surface receptors that then activate the operons that are responsible of progressing the bacteria's biofilm [9]

These operons then code for more signaling molecules or, more importantly, progress the staph biofilm life cycle, moving it into its maturation and dispersal phases. This information about QS induced staph aureus biofilms led to the hypothesis that a staph infection could be treated by finding some sort of chemical mimic for AIP in order to speed up the progression of the biofilm. The identification of the research gap opens a conversation about finding quorum sensing inducers in order to accelerate the dispersal of the Staphylococcus aureus biofilm. Finding this inducer helps prove this alternative approach of attack infection works, without having to follow the standard blueprint steps most use in discovering drug compounds.

\section{METHODOLOGY}

\section{In Silico Approach}

The staple experiment in testing this project would be to take strains of staph bacteria and saturate their environment with different molecules to see if any of them have an effect on the biofilm. Because of COVID restrictions along with financial and time restraints, doing a live experiment was not able to be accomplished for good reason. Working with and synthesizing these chemicals takes both time and energy that is not readily available for amateur experiments. Because of all these factors, I have decided to go through an in silico approach with this experiment. The reasoning behind this is that an experiment similar to this was done using an in silico approach and proved to be useful for scientists later when doing the laboratory experiment $[18,22]$. The in silico approach is the initial step that scientists take to assess the achievability of their experiment. In the context of the project at hand, This would consist of studying the binding mechanisms behind the agr $\mathrm{C}$ receptor and the different molecules that would be denoted as the hypothesized inducers. This process has two steps, first being the chemical screening of different molecules, then being the chemical docking. Both of these steps are required in order for the results of the in silico approach to be as accurate as possible.

\section{Chemical Screening}

The first step of the experiment is chemical screening, the identification of different molecules that could possibly act as the inducer. As described in the Principles of developmental genetics, "identify small molecules that produce a desired phenotype without prior knowledge" [16]. The goal for chemical screening is to be able to identify molecules that fit the characteristics for the needs of the experiment. These characteristics can be anything from looking for a specific atom in a compound to identifying a certain shape of the molecule. Most of this information can be found looking into previous studies published on chemical databases such as pubchem [21,22]. Chemical screening usually acts as qualitative data in the scheme of the in silico approach.

\section{Chemical Docking}

After distinguishing which molecules best fit the purpose of the experiment, the next step is modeling how the molecule interacts with the receptor in question. This is done through a process of chemical docking, in which the binding mechanisms of the reaction are quantified using the following formula [17]

$$
\begin{aligned}
E_{\mathrm{cle}}(r)= & \sum_{\text {bonds }} k_{\mathrm{b}}\left(r-r_{0, \mathrm{~b}}\right)^{2}+\sum_{\mathrm{UB}} k_{\mathrm{UB}}\left(S-S_{0, \mathrm{UB}}\right)^{2}+\sum_{\text {angle }} k_{\mathrm{a}}\left(\theta-\theta_{0, \mathrm{a}}\right)^{2} \\
& +\sum_{\text {dihedrals }} k_{\mathrm{d}, \mathrm{n}}\left(1+\cos \left(n \chi-\delta_{\mathrm{d}, \mathrm{n}}\right)\right)+\sum_{\text {impropers }} k_{i}\left(\psi-\psi_{0, i}\right)^{2} \\
& +\sum_{\mathrm{vdw}} \varepsilon_{i j}\left[\left(\frac{R_{\min , i j}}{r_{i j}}\right)^{12}-2\left(\frac{R_{\mathrm{min}, i j}}{r_{i j}}\right)^{6}\right]+\sum_{\text {electrostatic }} \frac{q_{i} q_{j}}{4 \pi \varepsilon_{0} r_{i j}}
\end{aligned}
$$


This was the original equation used to determine a numerical score, using a computer, of all the binding energies that are taken into account between two molecules. As mentioned before, this experiment uses a docking software from Schrodinger known as GLIDE. As one of the more accurate docking softwares, the GLIDE program uses a flexible docking to simulate precise representation of how binding would work in a true experiment[20]. The program, after taking into account all intermolecular forces, then gives out a number of how well the chemicals bind together. The steps for chemical docking includes creating a binding grid for the receptor model found on the protein database (PDB) [19] and then plugging each molecule that was chemically screened into said gird, and finally running the GLIDE program to get the GLIDE score for the binding mechanism, the closer the number is to 0 , the better binding interaction it has[20].

\section{Procedures}

Since in this scenario the target compound was a mimic of the AIP molecule, the inducers found through chemical screening were all chosen due to their similar properties to AIP. Other factors that were examined to identify the molecules include, how well they would hold up in anin vitro (live) environment and how cost effective it would be to synthesize these molecules. After examining the properties of AIP that wanted to be emulated, the 3 molecules that were chosen from the pubchem database [21] to be docked were: Homocysteine Thiolactone, Solonamide B, and Flustrabromine III. Further reasoning behind the choosing of these molecules will be discussed further in the qualitative data analysis. The next step would be to plug the receptor agrC into the Schrodinger modeling software using the PDB code 4BXI. Once the binding sites of the receptor are identified, the receptor grid can be created in order to dock the chosen molecules to agrC (Figure 4).

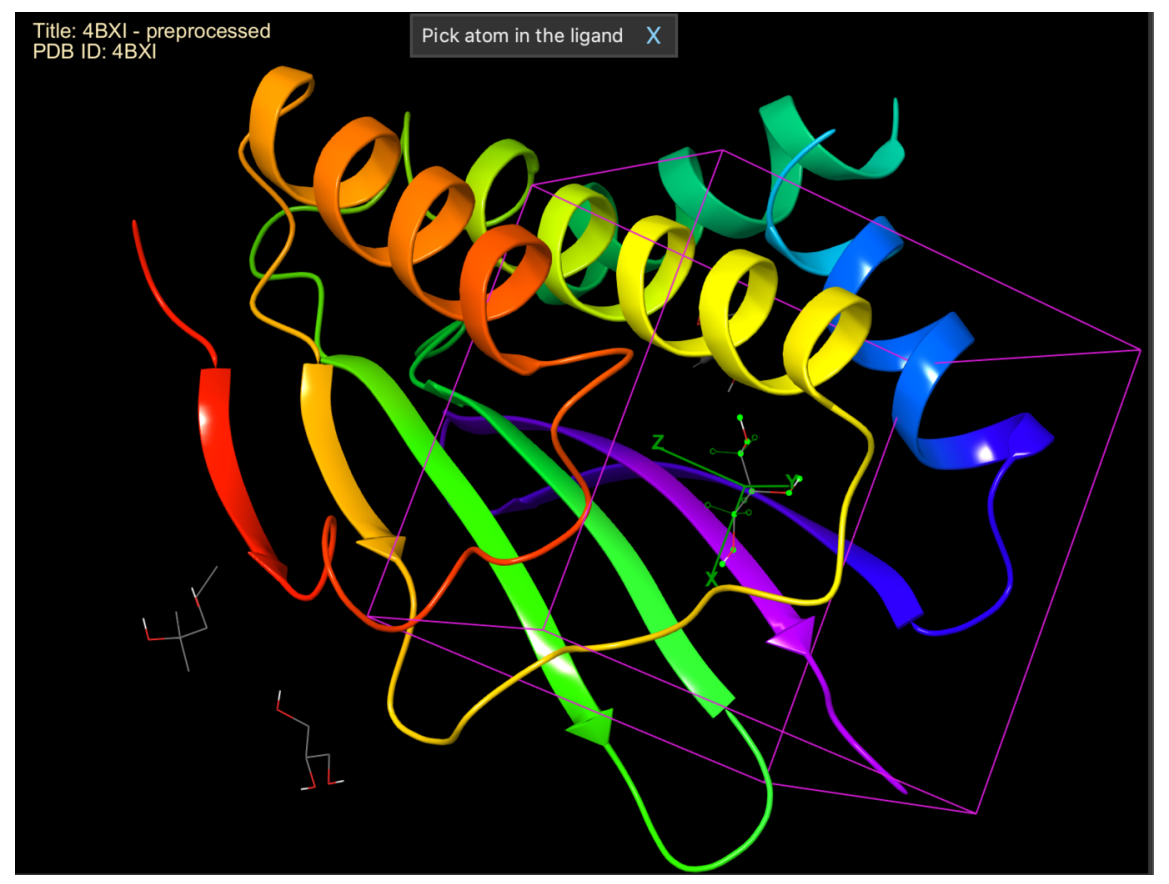

Figure 4: GLIDE grid generated for the agrC which will be used to evaluate the binding energies between receptor and appropriate ligands.

The final step to collect the quantitative data would be to dock the three molecules with agrC and find out which has the highest GLIDE score. After compiling the qualitative and quantitative data, the molecule that is the best QS inducer for $S$. aureus biofilms can be decided. In order to keep all other variables constant, the parameters to set up the receptor grid and GLIDE docking program are the same for all three compounds and can be found in table 1 and 2 of Appendix B respectively. 


\section{DATA AND ANALYSIS}

\section{Data from GLIDE Docking}

After all molecules were screened, modeled, and docked with GLIDE, scores were received from the program that were representative of the binding mechanics between each molecule with agr $\mathrm{C}$ binding site (figure 4). It's understood that the less energy the binding gives off, or the closer the GLIDE value is to 0, the more affinity the receptor has to that molecule. Another quality that seemed to have an advantage is the relative size of the molecule. Molecules of small size seem to have lower binding energies and, hypothetically, will have a larger effect on the QS mechanisms of the bacteria. The values are compiled in the data table and graph below. Each molecule will be reviewed for both its qualitative features and how well it binds with the receptor according to their individual GLIDE scores. The 2-D models for each molecule can be found in Appendix A.

GLIDE Scores for Tested Molecules

\begin{tabular}{lllll}
\hline Experiment & Molecule Name & Pubchem Code & Docked PDB & Docking Score (kcal \\
\hline Control & AIP & AIP 3.sdf & 4BXI (agrC binding mechanism) & -7.661 \\
Molecule 1 & Homocysteine Thiolactone & hcy thio.sdf & 4BXI & -3.35 \\
Molecule 2 & Solonamide B & so.sdf & 4BXI & no poses \\
Molecule 3 & Flustrabromine III & indol.sdf & 4BXI & -3.54 \\
\hline
\end{tabular}

\section{GLIDE Docking Scores}

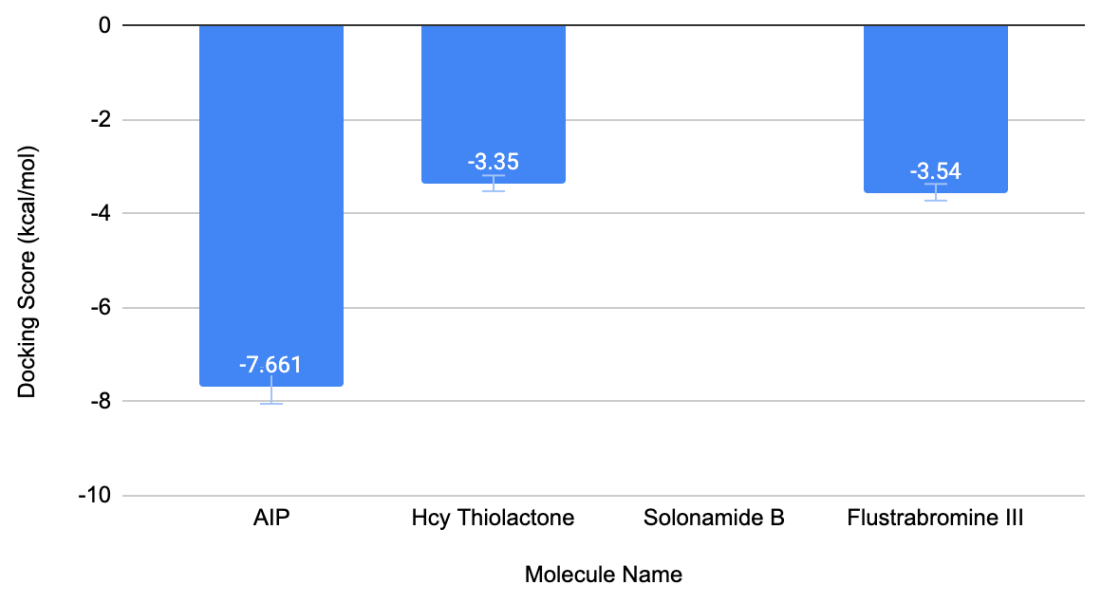

\section{AIP}

As the control molecule, AIP (see Table 1) is a relatively large, high energy peptide that has multiple key features that allow it to bond with the agrC . Some defining features behind the peptide are the ring structures that are labeled as thiolactone linkages. Chemical screening done by Princeton University's Boyuan Wang, shows a stabilizing ammonia chain to the thiolactone seen in the button right of figure 5 [23]. This allows the molecule to release higher energies when attached to the agrC binding site (figure 5). It should also be noted that AIP is a naturally produced compound by Staphylococcus cells 


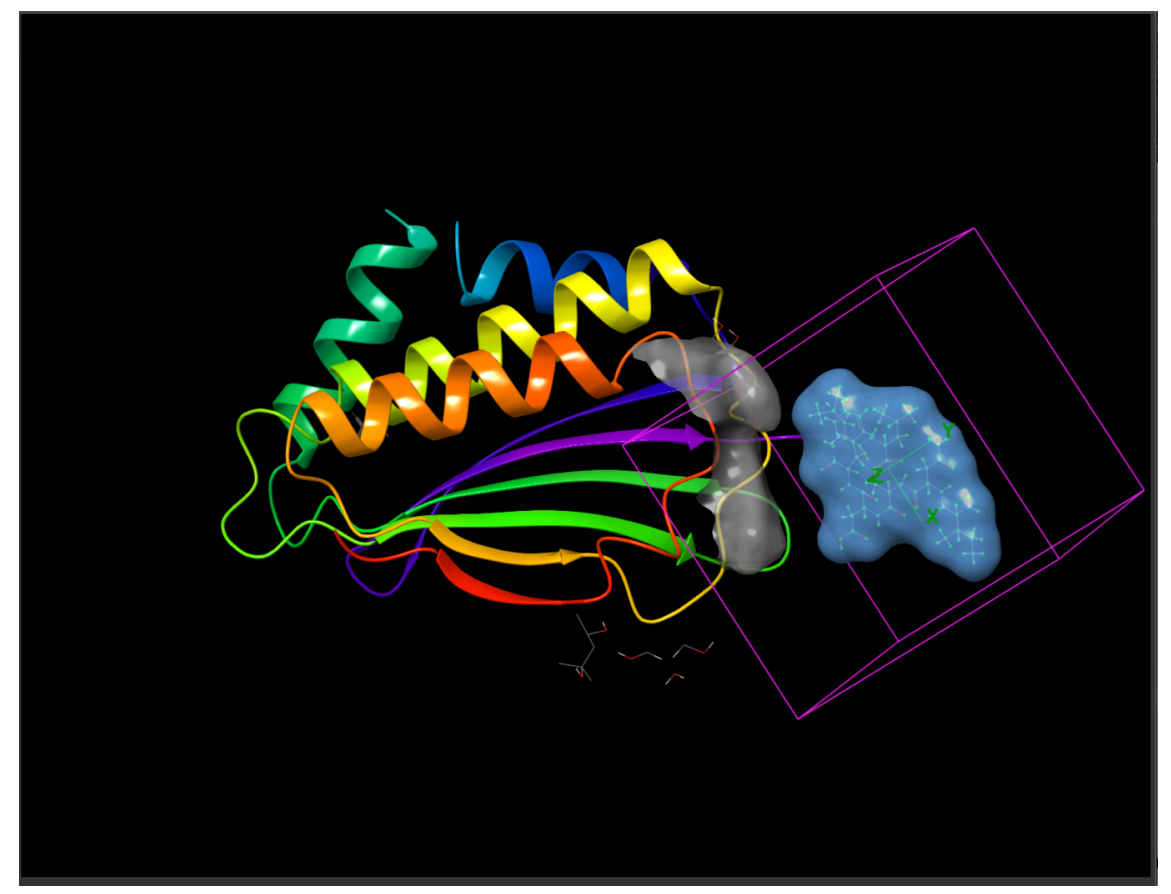

Figure 5: Docking grid generation of AIP molecule using Schrodinger[20]

After creating the binding model in Schrodinger and docking the control compound, the GLIDE score received was $-7.661 \mathrm{kcal} / \mathrm{mol}$. Being that this is the control molecule and judging by the relatively large size of the peptide, this was the predicted score for AIP. The larger size adds unnecessary repelling charges between AIP and agr, but are seen as vital for the compound's stabilization. From this control module, there is a set basis for what defining binding points should be looked for when choosing the other screened compounds. It also gives lots of gap to work in between as getting a lower GLIDE score than AIP will mean the molecule can act as a competitive inducer to AIP in an in vitrosetting.

\section{Homocysteine Thiolactone}

Hcy Thiolactone is a small molecule with a molecular weight of only about $117.7 \mathrm{~g} / \mathrm{mol}[21]$. Thiolactone is made up of a few carbon compounds with some oxygen chains attached. The defining portion that adds the overall charge of the molecule would be the sulfur seen on the bottom of figure 6 . From the study done by Wang, it was known that thiolactone linkages were a major component to the AIP makeup. From this information, it was hypothesized that thiolactone was also the main binding component to the agr-AIP system. In order to test this, thiolactone was isolated and docked with agr $\mathrm{C}$ to see how well it would fit together. 


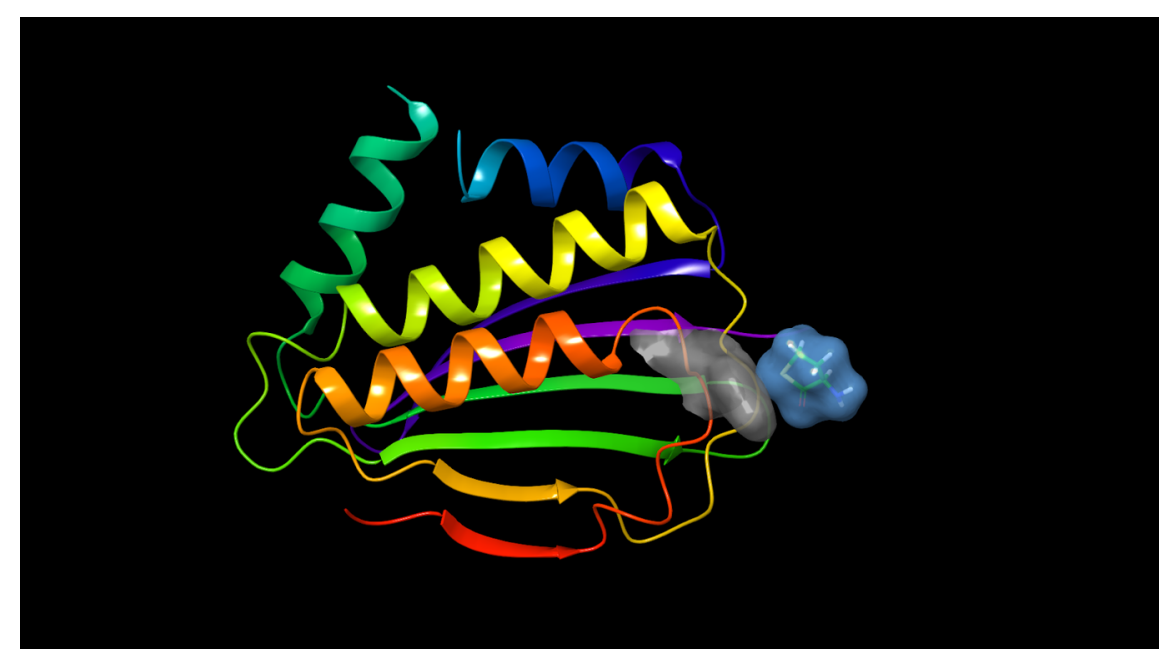

Figure 6: Grid Docking of Hcy Thiolactone with agrC receptor binding site[20,22]

The isolated thiolactone received a score of $-3.35 \mathrm{kcal} / \mathrm{mol}$, significantly lower than its parent counterpart. This is mainly due to its smaller size and as well as some of its properties being highlighted more when not covered by the rest of the carbon chains seen on AIP. Although the molecule, in theory, would work very well as an inducer, during the chemical screening process it was understood that isolating and synthesizing thiolactone itself would be a strenuous process. There are specific variants of thiolactone that have a methionine peptide attached to it but have shown to have severe repercussions on the human body [24]. Further research on the screening of this particular type of thiolactone before it can be put to practical use.

\section{Solonamide B}

As a molecule designed to mimic peptides, or peptidomimetic, the Solonamide family of molecules have shown promising results when used in experimentation with other strains of bacteria. In fact, a study done by the University of Copenhagen has shown that Solonamide B has had an inhibiting effect on the QS mechanism of altered strains of S aureus bacteria[25]. It is still unclear, however, on whether or not Solonamide is acting as a competitive inhibitor or was completely disturbing the receptor's chemical makeup, either way docking the molecule would give a better understanding on why the interactions occur as it does.

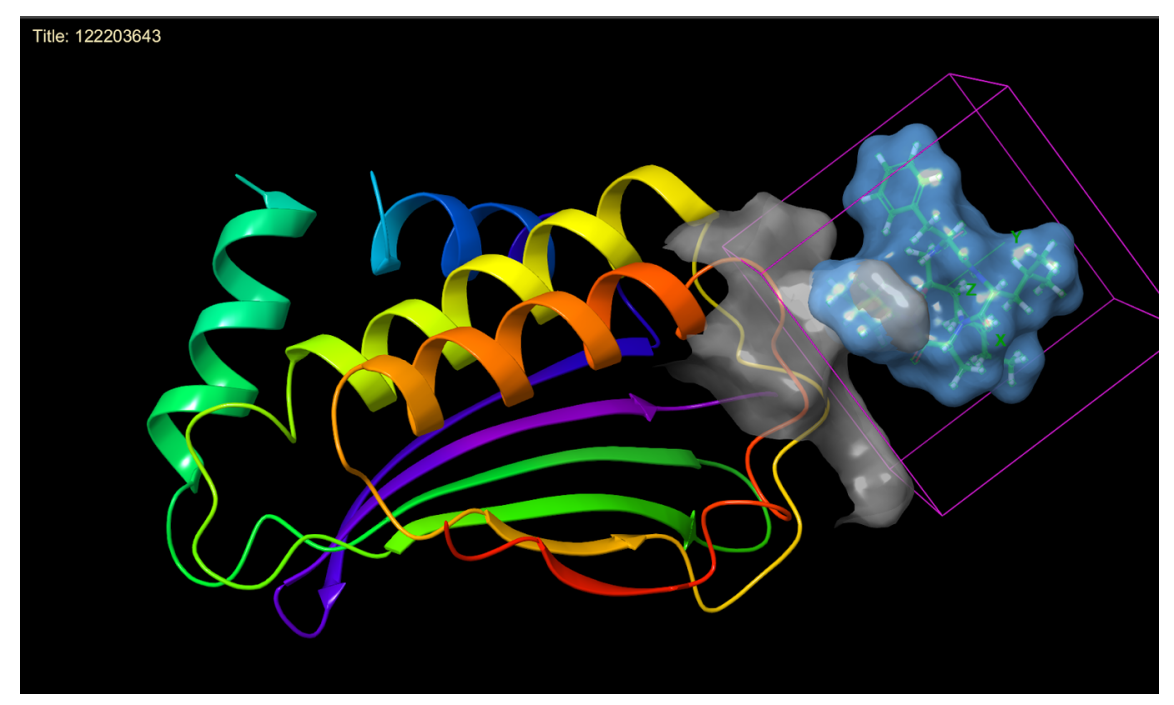




\section{Figure 7: agr-solonamide B docking complex on schrodinger}

After docking the solonamide $\mathrm{B}$ to agr $\mathrm{C}$ it was found to have no poses or common binding areas. This might be due to the fact of the sheer size of solonamide B, which the Schrodinger software automatically bound to the largest binding site on the agrC model for good reason (figure 7). Some other reasons behind the null docking score could be because of some experimental parameters chosen for the experiment. The Solonamide strain used in the experiment was described to be a linear peptidomimetic and the model used in GLIDE has multiple rings and has quite a few highly reactive chemicals on the end of them [25]. This model of Solonamide is also found naturally in gram negative bacteria so its stable rings may be an evolutionary trait that would require a synthesizing alteration if this project were to pursue this molecule. Creating a variant by removing the carbon rings might give a nicer answer when docked.

\section{Flustrabromine III}

The final molecule that was screened and chosen to be docked with agr was an indole variant known as flustrabromine. This compound is an indolic flustramine, a molecule isolated from the secondary metabolites of an invertebrate sea creature known as Flustra foliacea[26]. The process in which these nodules are created is inexpensive and easy to do, making the molecule easy to access for anin vitro setting. After isolating the base indole molecule from the foliacea, a bromine is added onto the end of its hexagonal structure in order for the compound to be more reactive (see table 1). As one of the few inducers with a naturally occurring bromine, AIP's chemical screening test also hypothesized that attached bromine might hold a significant factor on it's binding mechanism with agr C. In order to see if the bromine has an effect on the binding mechanism, Flustrabromine III was docked with agr $\mathrm{C}$ with an emphasis on its bromine structure (figure 8).

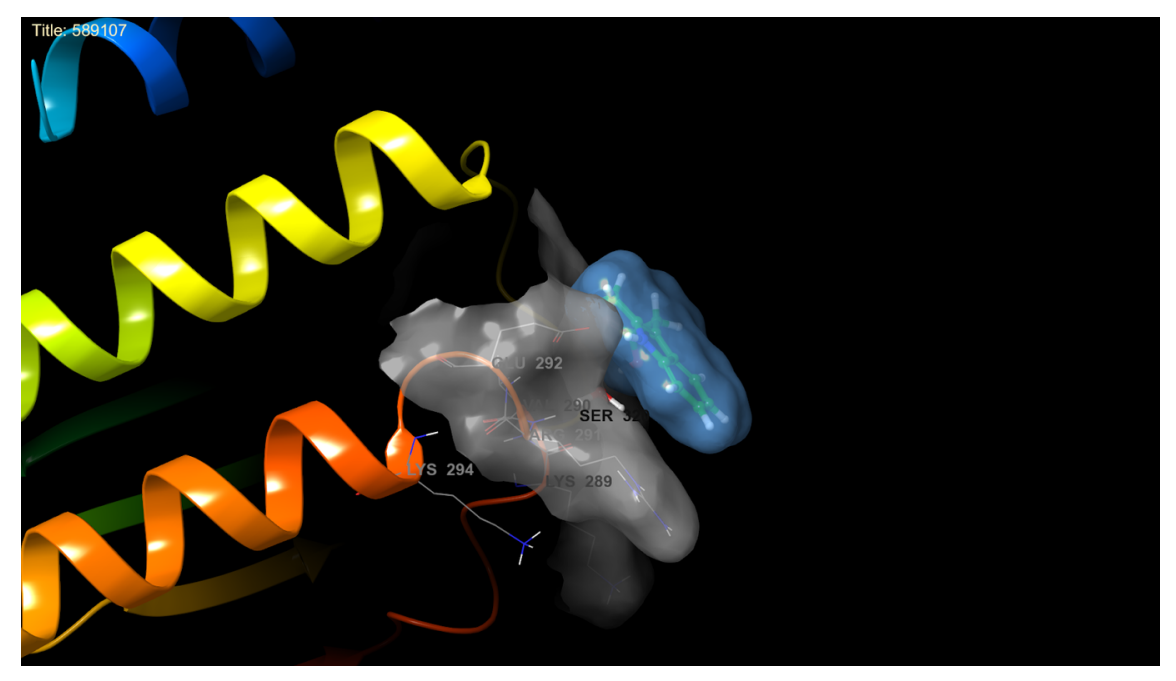

Figure 8: post GLIDE docking analysis of flustrabromine III- agr C shows a bonding between the bromine end of the molecule to the GLU 292 section of the agr binding site.

Flustrabromine earned a score of $-3.54 \mathrm{kcal} / \mathrm{mol}$ which is the second highest recorded in the quantitative data. This shows that the bromine has some sort of an effect on the agr system, in vitroexperimentation will give more insight into whether or not this molecule actually does accelerate the growth of the staph biofilm. Since the flustra bromine molecule is much easier to synthesize and it's qualitative features are much more favorable compared to the other molecules making it the logically best inducer for the goals of this project.

\section{CONCLUSION}

\section{Implications and Limitations}


After all data was collected and each molecule's chemical screening and docking results were examined, it was decided that flustrabromine III was the best overall synthetic inducer for accelerating the growth of $S$. aureus biofilms. Although from a quantitative lens, the isolated thiolactone had a higher GLIDE score, filtering in the qualitative facets that were pulled from the chemical screenings of each molecule shows that flustrabromine has better reactive measures than the thiolactone and is much easier to synthesize and use as a drug $[24,26]$. The ability to synthesize a molecule plays a large factor on the feasibility to use that compound in future experimentation or even the distribution of the compound as medicine. If a molecule takes more effort and money to create, it is not apt for practical use. Another factor in play that gave an advantage to Flustrabromine was the predicted effect each molecule would have on the human body. The parameters chosen for the chemical docking portion of this project were chosen to emulate the prime conditions for staph bacteria, which happen to be resemblant of the conditions in the human body. It was seen through previous research that in this environment thiolactone molecules are susceptible to alterations in their make-up and can lead to the creation of carcinogenic substances. Because of this hypothesized toxic characteristics thiolactone was seen out of the scope of practical use.

The results of this experiment are hindered by the extent at which the quantitative data was explored. Due to time constraints and sheer complexity of chemical docking, the level of analysis behind the GLIDE results hardly scratches the surface of all the information that can be pulled from them. The different types of docking expand on the sophistication of the results, ranging from rigid docking (the most basic results) to flexible docking (the most accurate results) [18]. In order to find a middle ground for the complexity of the data, this project encompasses an induced fit docking, which means the ligand gives semi-accurate results without having to understand everything about python script in bioinformatics. However, this decision, along with a partial lack of understanding of the results, created room for potential error. With the docking score difference between flustrabromine and thiolactone being so close, this misconception can scale up when progressing further into an in vitro experiment. If there were more time for experimentation, each GLIDE result could be broken down into different poses, or variations in which the molecule can bond with agr-C. This then can be taken through a statistical analysis where one can claim the molecule with the more poses with a docking score equal to or higher than the average score (the one documented in the data) is the molecule that is more likely to bind with agrC. With such an open ended methodology in this project there are multiple factors that could have altered the data in a small way, but none are significant enough to warrant a change in the conclusion that flustrabromine is the qualitatively superior inducer for agr C.

\section{Future Research}

As mentioned throughout this paper, this experiment was a basis for the true lab based project or in vitro approach. Much of the methods in this project are based on theory which makes it difficult to assess if the molecules will do exactly what they were predicted to do. Nonetheless, The dockings do confirm that these molecules will bind to the agr binding site but whether or not it can start off the QS mechanism is something that can be explored either through expanding the docking environment or through a laboratory experiment. This project ultimately accomplished its goal of furthering the understanding behind how QS mechanisms can be manipulated using different compounds. It also gives a good insight on how chemical screening and docking can be used in alternative methods rather than the standard procedures in which they are normally seen. Using computers in molecular biology is a revolutionary topic and combining those resources with the up and coming research into QS mechanisms allows greater discernment on how infections caused by bacteria, such as $S$. aureus, can be treated with alternative medicines found through drug discovery procedures such as the one used in this project. Hopefully, with the progression of quorum sensing research, this project can use its novel approach to explore more questions revolving around the frontier of precision medicine and drug discoveries

\section{References}

1. BL; MMBB. Quorum sensing in bacteria [Internet]. Annual review of microbiology. U.S. National Library of Medicine; [cited 2021Apr28]. Available from: https://pubmed.ncbi.nlm.nih.gov/11544353/\#: :text=Quorum\%20 sensing\%20is\%20the\%20 reg- 
ulation,a\%20function\%20of\%20cell\%20 density.

2. Li Z, Nair SK. Quorum sensing: how bacteria can coordinate activity and synchronize their response to external signals? [Internet]. Protein science : a publication of the Protein Society. Wiley Subscription Services, Inc., A Wiley Company; 2012 [cited 2021Apr28]. Available from: https://www.ncbi.nlm.nih.gov/pmc/articles/PMC3526984/

3. González JE, Keshavan ND. Messing with Bacterial Quorum Sensing [Internet]. Microbiology and Molecular Biology Reviews. American Society for Microbiology; 2006 [cited 2021Apr28]. Available from: https://mmbr.asm.org/content/70/4/859\#: : :text=Quorum\%20Sensing\%20in\%20Gram\%2DNegative,reach\%20a\%20 high $\% 20$ population $\% 20$ density.

4. Cunningham A. [Internet]. Virulence. [cited 2021Apr28]. Available from: https://www.cs.montana.edu/webworks/projects/stevesbook/contents/chapters/chapter002/section004/blue/page002.htr

5. Kırmusaoğlu S. Staphylococcal Biofilms: Pathogenicity, Mechanism and Regulation of Biofilm Formation by Quorum-Sensing System and Antibiotic Resistance Mechanisms of Biofilm-Embedded Microorganisms [Internet]. IntechOpen. IntechOpen; 2016 [cited 2021Apr28]. Available from:https://www.intechopen.com/books/microbial-biofilms-importanceand-applications/staphylococcal-biofilms-pathogenicity-mechanism-and-regulation-of-biofilm-formationby-quorum-sensing

6. Phillips PL, Schultz GS. Molecular Mechanisms of Biofilm Infection: Biofilm Virulence Factors [Internet]. Advances in wound care. Mary Ann Liebert, Inc.; 2012 [cited 2021Apr28]. Available from:https://www.ncbi.nlm.nih.gov/pmc/articles/PMC3839007/

7. $\mathrm{PhD}$ KS. Gram Positive vs Gram Negative [Internet]. from Technology Networks. Technology Networks; 2020 [cited 2021Apr28]. Available from:https://www.technologynetworks.com/immunology/articles/gram-positive-vs-gram-negative323007

8. Whitehead NA, Barnard AML, Slater H, Simpson NJL, Salmond GPC. Quorum-sensing in Gramnegative bacteria [Internet]. OUP Academic. Oxford University Press; 2001 [cited 2021Apr28]. Available from: https://academic.oup.com/femsre/article/25/4/365/524818

9. Quorum Sensing in Staphylococcus aureus Infections [Internet]. microbewiki. 2007 [cited 2021Apr28]. Available from: https://microbewiki.kenyon.edu/index.php/Quorum_Sensing_in_Staphylococcus_aureus_Infections

10. Sankar Ganesh P., Ravishankar Rai V. (2018) Alternative Strategies to Regulate Quorum Sensing and Biofilm Formation of Pathogenic Pseudomonas by Quorum Sensing Inhibitors of Diverse Origins. In: Kalia V. (eds) Biotechnological Applications of Quorum Sensing Inhibitors. Springer, Singapore.https://doi.org/10.1007/978-981-10-9026-4_3

11. Whitehead NA, Barnard AML, Slater H, Simpson NJL, Salmond GPC. Quorum-sensing in Gramnegative bacteria [Internet]. OUP Academic. Oxford University Press; 2001 [cited 2021Apr28]. Available from: https://academic.oup.com/femsre/article/25/4/365/524818

12. Matsumoto K, Takeuchi S, Uehara Y, Matsushita M, Arise K, Morimoto N, et al. Transmission of methicillin-resistant Staphylococcus aureus in an acute care hospital in Japan [Internet]. Journal of general and family medicine. John Wiley and Sons Inc.; 2018 [cited 2021Apr28]. Available from: https://www.ncbi.nlm.nih.gov/pmc/articles/PMC6321822/

13. Paluch E, Rewak-Soroczyńska J, Jędrusik I, Mazurkiewicz E, Jermakow K. Prevention of biofilm formation by quorum quenching [Internet]. Applied Microbiology and Biotechnology. Springer Berlin Heidelberg; 2020 [cited 2021Apr28]. Available from:https://link.springer.com/article/10.1007/s00253020-10349-w\#cite

14. Li Y-H, Tian X. Quorum sensing and bacterial social interactions in biofilms [Internet]. Sensors (Basel, Switzerland). Molecular Diversity Preservation International (MDPI); 2012 [cited 2021Apr28]. Available from:https://www.ncbi.nlm.nih.gov/pmc/articles/PMC3376616/

15. Salmaso V, Moro S. Bridging Molecular Docking to Molecular Dynamics in Exploring Ligand-Protein Recognition Process: An Overview [Internet]. Frontiers. Frontiers; 2018 [cited 2021May16]. Available from: https://www.frontiersin.org/articles/10.3389/fphar.2018.00923/full\#: : :text=.\%2C\%202015 
16. Chemical Screening [Internet]. Chemical Screening - an overview | ScienceDirect Topics. [cited 2021May16]. Available from:https://www.sciencedirect.com/topics/biochemistry-genetics-andmolecular-biology/chemical-screening\#: : text=Chemical\%20screening\%20is\%20a\%20central\%20component\%20of\%20m

17. MacKerell AD;Bashford D;Bellott M;Dunbrack RL;Evanseck JD;Field MJ;Fischer S;Gao J;Guo H;Ha S;Joseph-McCarthy D;Kuchnir L;Kuczera K;Lau FT;Mattos C;Michnick S;Ngo T;Nguyen DT;Prodhom B;Reiher WE;Roux B;Schlenkrich M;Smith JC;Stote R;Straub J;Watanabe M;Wi. All-atom empirical potential for molecular modeling and dynamics studies of proteins [Internet]. The journal of physical chemistry. B. U.S. National Library of Medicine; [cited 2021May16]. Available from: https://pubmed.ncbi.nlm.nih.gov/24889800/

18. Barriuso J, Martínez MJ. In Silico Analysis of the Quorum Sensing Metagenome in Environmental Biofilm Samples [Internet]. Frontiers in microbiology. Frontiers Media S.A.; 2018 [cited 2021Apr28]. Available from:https://www.ncbi.nlm.nih.gov/pmc/articles/PMC6000730/

19. Bank RCSBPD. Protein Database [Internet]. RCSB PDB. [cited 2021May16]. Available from:https://www.rcsb.org/

20. Robinson K. Schrodinger Modeling Software [Internet]. Citations | Schrödinger. 2016 [cited 2021May16]. Available from:https://www.schrodinger.com/citations

21. PubChem [Internet]. National Center for Biotechnology Information. PubChem Compound Database. U.S. National Library of Medicine; 1998 [cited 2021May16]. Available from: https://pubchem.ncbi.nlm.nih.gov/

22. Pagadala NS, Syed K, Tuszynski J. Software for molecular docking: a review [Internet]. Biophysical reviews. Springer Berlin Heidelberg; 2017 [cited 2021May16]. Available from: https://www.ncbi.nlm.nih.gov/pmc/articles/PMC5425816/

23. Wang B, Zhao A, Novick RP, Muir TW. Key driving forces in the biosynthesis of autoinducing peptides required for staphylococcal virulence [Internet]. PNAS. National Academy of Sciences; 2015 [cited 2021May16]. Available from: https://www.pnas.org/content/112/34/10679.short\#: : :text=Autoinducing\%20peptide\%20(AIP)\%2C\%20a,energy\%20 input\%20from\%20ATP\%20 hydrolysis.

24. Homocysteine Thiolactone [Internet]. Homocysteine Thiolactone - an overview | ScienceDirect Topics. [cited 2021May16]. Available from:https://www.sciencedirect.com/topics/pharmacology-toxicology-andpharmaceutical-science/homocysteine-thiolactone

25. Karathanasi G, Bojer MS, Baldry M, Johannessen BA, Wolff S, Greco I, et al. Linear peptidomimetics as potent antagonists of Staphylococcus aureus agr quorum sensing [Internet]. Nature News. Nature Publishing Group; 2018 [cited 2021May16]. Available from:https://www.nature.com/articles/s41598018-21951-4\#Sec3

26. Worthington RJ, Richards JJ, Melander C. Small molecule control of bacterial biofilms [Internet]. Organic \& biomolecular chemistry. U.S. National Library of Medicine; 2012 [cited 2021May16]. Available from:https://www.ncbi.nlm.nih.gov/pmc/articles/PMC3431441/

\section{Additional Sources}

Lyon GJ, Mayville P, Muir TW, Novick RP. Rational design of a global inhibitor of the virulence response in Staphylococcus aureus, based in part on localization of the site of inhibition to the receptor-histidine kinase, AgrC [Internet]. PNAS. National Academy of Sciences; 2000 [cited 2021Apr28]. Available from: https://www.pnas.org/content/97/24/13330

Zhu, K.; Day, T.; Warshaviak, D.; Murrett, C.; Friesner, R.; Pearlman, D., "Antibody structure determination using a combination of homology modeling, energy-based refinement, and loop prediction," Proteins, 2014, 82(8), 1646-1655

Salam, N.K.; Adzhigirey, M.; Sherman, W.; Pearlman, D.A., "Structure-based approach to the prediction of disulfide bonds in proteins," Protein Eng Des Sel, 2014, 27(10), 365-74

Beard, H.; Cholleti, A.; Pearlman, D.; Sherman, W.; Loving, K.A., "Applying Physics-Based Scoring to Calculate Free Energies of Binding for Single Amino Acid Mutations in Protein-Protein Complexes,"PLoS 
ONE,2013, 8(12), e82849

PubChem [Internet]. National Center for Biotechnology Information. PubChem Compound Database. U.S. National Library of Medicine; 2012 [cited 2021Apr28]. Available from: https://pubchem.ncbi.nlm.nih.gov/

Karathanasi G, Bojer MS, Baldry M, Johannessen BA, Wolff S, Greco I, et al. Linear peptidomimetics as potent antagonists of Staphylococcus aureus agr quorum sensing [Internet]. Nature News. Nature Publishing Group; 2018 [cited 2021Apr28]. Available from:https://www.nature.com/articles/s41598-018-21951-4\#Sec3

Worthington RJ, Richards JJ, Melander C. Small molecule control of bacterial biofilms [Internet]. Organic \& biomolecular chemistry. U.S. National Library of Medicine; 2012 [cited 2021Apr28]. Available from: https://www.ncbi.nlm.nih.gov/pmc/articles/PMC3431441/

Jakubowski H. Homocysteine Thiolactone: Metabolic Origin and Protein Homocysteinylation in Humans [Internet]. OUP Academic. Oxford University Press; 2000 [cited 2021Apr28]. Available from: https://academic.oup.com/jn/article/130/2/377S/4686422

Zhang M, Ding S. Chemical Screening [Internet]. Chemical Screening - an overview | ScienceDirect Topics. 2019 [cited 2021Apr28]. Available from:https://www.sciencedirect.com/topics/biochemistry-geneticsand-molecular-biology/chemical-screenin

\section{Acknowledgments}

Dr. C.N. Ramchand (CEO of Saksin lifesciences) for initial guidance and recommended analysis of results

Professor Krishna Kumar PhD (professor in bioinformatics at IIT Delhi) for recommendation of the Schrodinger LLC modeling software for more accurate analysis

Dr. Devi Santosh (lead biochemistry analyst at Medgenome Inc.) for detailed review of the research methodology and suggesting further extension of the original research scope

\section{Appendix A}

Appendix A below shows the 2-D models of each molecule from Pubchem that were chosen in the chemical screening process. Their 3-D constructions were then used in the Schrodinger docking program. These figures are here to provide a look at the basis of each molecule and the similarities they may have with the control AIP compound.

\section{2-D Molecular Constructions}

\section{Hosted file}

Flustrabromine III.png available at https://authorea.com/users/423562/articles/528979simulating-drug-discoveries

\section{Hosted file}

Solanamide B.png available at https://authorea.com/users/423562/articles/528979-simulatingdrug-discoveries

\section{Hosted file}

Homocysteine thiolactone.png available at https://authorea.com/users/423562/articles/528979simulating-drug-discoveries

\section{Hosted file}

AIP.png available at https://authorea.com/users/423562/articles/528979-simulating-drugdiscoveries

Appendix B 
Table 1 shows the parameters used to establish the GLIDE receptor grid. These parameters were modeled after the optimal conditions for the $S$. aureus biofilms and were kept constant when docking every single molecule. These parameters can be altered in future research to see how the docking scores will be altered based on the conditions set in Schrodinger.

\section{Table 1: Grid Receptor Generation Parameters}

\section{Receptor}

\begin{tabular}{ll}
\hline Define receptor & on \\
Van der Waals radius scaling factor & 1.0 \\
Partial charge cutoff & .25 \\
Use input partial charges & off \\
Generate grid suitable for peptide docking & on \\
Site & Site \\
Docked ligand is confined to the enclosing box & on \\
Center & Center of workspace ligand \\
Docked ligand size & 15 \\
Constraints & Constraints \\
Show positional markers & on \\
Label positions & on \\
H-bonds/Metal bonds & 0 \\
Metal coordination & 0 \\
Rotatable groups & none \\
Label excluded volumes & on \\
Postmortem Parameter Assignments & Postmortem Parameter Assignments \\
VdW radius & 1.0 \\
Receptor setup location & $(101,15,15,15,1)$ \\
DOCKMAIN grid & $(40,40,40)$ \\
\hline
\end{tabular}

Table 2 is a culmination of all important parameters set for the GLIDE chemical docking software. These parameters were built off those set in the receptor grid (seen in table 1) and remained the same for all docked molecules with agr-C.

\section{Table 2: Induced Fit Ligand Docking Parameters}

\begin{tabular}{ll}
\hline Receptor Grid & From file \\
\hline File name & glide-grid_ $7 \_$subjobs_log.maegz \\
Show grid boxes & on \\
Display receptor & off \\
Ligands & Ligands \\
Use ligands from & Phase Database \\
Range & $1-455$ \\
Use input partial charges & off \\
Ligand docking and scoring limit & 500 atoms and 1000 rotatable bonds \\
Scaling factor of van der Waal radii & .8 \\
Partial charge cutoff & .15 \\
Settings & Settings \\
Precision & SP-peptide \\
Ligand Sampling & Flexible \\
Sample nitrogen inversions & on \\
Sample ring conformations & on
\end{tabular}




\begin{tabular}{ll}
\hline Receptor Grid & From file \\
\hline Sample macrocycle using Prime Non-macrocyclic ligands & off \\
Include Input ring conformations & off \\
Bias sampling of torisons for: & All predefined functional groups \\
Add epik state penalties to docking score & on \\
Reward intermolecular hydrogen bonds & off \\
Enhance planarity of conjugated pi groups & off \\
Energy window for ring sampling & $2.5 \mathrm{kcal} / \mathrm{mol}$ \\
Include aromatic H-bonds as donors & off \\
Distance dependent dielectric constant & 2.0 \\
Constraints & Constraints \\
Use core pattern comparison & on \\
Restrict docking to a reference position & on \\
Tolerance & .10 \\
Define core & Workspace ligand \\
Maximum common substructures & on \\
All heavy atoms are core atoms & off \\
SMARTS pattern & off \\
Pick RMSD subset atoms & off \\
Show grid based markers & on \\
Apply shape constraints & off \\
Define workspace ligand & Entry.id 12 AND mol.entry 1 \\
Torsional patterns & none \\
Constrain all torisons & off \\
Output & Output \\
File type & Pose view file (includes receptor) \\
Limit the number of poses & off \\
Most poses per ligand & infinite \\
Perform post docking minimization & on \\
Number of poses per ligand to include & 5 \\
Write per-residue interaction scores & off \\
Compute RMSD to input ligand geometries & on \\
\hline
\end{tabular}

